\title{
Inhibitory Effects of Stephania tetrandra S. Moore on Free Radical- Induced Lysis of Rat Red Blood Cells
}

\author{
Nobuyasu SekiYa, ${ }^{* a}$ Hiroaki Hikiami, ${ }^{a}$ Koichi Yokoyama, ${ }^{a}$ Kazufumi Kouta,${ }^{a}$ Iwao Sakakibara, ${ }^{b}$ \\ Yutaka SHIMADA, ${ }^{a, c}$ and Katsutoshi TERASAWA ${ }^{c}$ \\ ${ }^{a}$ Department of Japanese Oriental Medicine, Faculty of Medicine, Toyama Medical and Pharmaceutical University; 2630 \\ Sugitani, Toyama 930-0194, Japan: ${ }^{b}$ Pharmacognosy \& Medicinal Resources Laboratory, Tsumura \& Co.; 3586 \\ Yoshiwara, Amimachi, Ibaraki 300-1192, Japan: and ${ }^{c} 21$ st Century COE Program, Toyama Medical and Pharmaceutical \\ University; 2630 Sugitani, Toyama 930-0194, Japan. \\ Received October 14, 2004; accepted January 24, 2005; published online January 27, 2005
}

Crude preparations of Stephania tetrandra S. Moore (ST), a traditional herbal medicine, have been used safely for arthritis and silicosis in China. In this study, we demonstrated that ST in vitro protects red blood cells from 2,2-azo-bis (2-amidinopropane) dihydrochloride (AAPH)-induced hemolysis. The inhibitory effect was dose-dependent at concentrations of 10 to $1000 \mu \mathrm{g} / \mathrm{ml}$. Moreover, tests were carried out to identify the main ingredient of ST that exerts a scavenging effect on free-radicals. Three representative alkaloids, tetrandrine, fangchinoline, and cyclanoline, isolated from ST, were found to have inhibitory activities against AAPH-induced lysis of red blood cells (RBC). Furthermore, the ingestion of $200 \mathrm{mg}$ of ST extract was associated with a significant increase in free-radical scavenging effect of plasma in rats. These results suggest that $\mathrm{ST}$ as antioxidant inhibits AAPH-induced hemolysis of RBC both in vitro and in vivo.

Key words antioxidative effect; reactive oxygen specie; Stephania tetrandra

Stephania tetrandra (ST) has been used as an analgesic drug for arthritis for thousands of years in China. ST has been reported to have anti-inflammatory ${ }^{1,2)}$ and immunosuppressive $^{3)}$ properties in vitro and in experimental animals. This study was performed to clarify the role of ST from an antioxidative point of view.

In the present study, we examined the effects of ST on scavenging activities of reactive oxygen species (ROS) by means of electron spin resonance (ESR) method, and on red blood cell (RBC) hemolysis induced by an azo free-radical initiator in healthy volunteers. The ESR method was used for the measurement of $\mathrm{O}_{2}^{-} \cdot$ and $\mathrm{HO} \cdot$ scavenging activities of ST. RBC hemolysis was used for determination of the antioxidative effect of ST, and the influence of ST on RBCs, which have the most important role in hemorrheology.

Furthermore, we hypothesized that ingestion of ST extract (ST.E) would further strengthen the antioxidative effect of plasma, which already has a function of reducing the free radical-induced tissue damage in cerebrovascular disease and ischemic heart disease, if the responsible compounds in ST could be absorbed and circulated in blood. Therefore, the present study examined the effect on plasma antioxidative activity after a gavage-dose of ST.E in rats.

\section{MATERIALS AND METHODS}

Preparation of Drugs ST.E was prepared from Stepha- nia tetrandra (Chinese origin) purchased commercially (Uchida Wakanyaku Co. Ltd., Tokyo, Japan). The extract was obtained by boiling the herb in water for $50 \mathrm{~min}$ and then freeze-drying into a resultant powder. We obtained $10.67 \mathrm{~g}$ of extract from $100 \mathrm{~g}$ of raw material.

This powder extract was dissolved in phosphate buffered saline (PBS, $\mathrm{pH}=7.4$ ).

Reagents Tetrandrine (TET), fangchinoline (FAN) and cyclanoline (CYC) were the products of Tsumura \& Co. (Tokyo, Japan). Their contents in ST.E were $2 \%, 1 \%$ and $1 \%$, respectively. The chemical structures of TET, FAN and CYC are illustrated in Fig. 1. The purity of each compound was examined by HPLC using a photodiode array detector.

ESR Assay for $\mathrm{O}_{2}^{-} \cdot$ and $\mathrm{HO} \cdot$ Scavenging Activities of ST.E Measurement of $\mathrm{O}_{2}^{-} \cdot$ and $\mathrm{HO} \cdot$ scavenging activities was performed as described previously ${ }^{4}$ with slight modification. ST.E was diluted to $0.2,2.0$, and $20.0 \mathrm{mg} / \mathrm{ml}$ with PBS. $\mathrm{O}_{2}^{-} \cdot$ and $\mathrm{HO} \cdot$ scavenging activities of each sample $(50 \mu \mathrm{l})$ were assessed by electron spin resonance (ESR) technique ${ }^{5}$ and then $\mathrm{IC}_{50}$ (Inhibition Concentration 50\%) was calculated.

In this experiment, $\mathrm{O}_{2}^{-} \cdot$ was generated from a hypoxanthine (HPX)-xanthine oxidase (XOD) reaction system in PBS. $^{6}$ Briefly, $15 \mu \mathrm{l}$ of $9.2 \mathrm{M}$ 5,5-dimethyl-1-pyrroline $\mathrm{N}$ oxide (DMPO) (LABOTEC, Tokyo, Japan), $50 \mu \mathrm{l}$ of $2 \mathrm{~mm}$ HPX (Sigma, St. Louis, MO, U.S.A.), $35 \mu 1$ of $5.5 \mathrm{~mm}$ diethylenetriamine- $N, N, N^{\prime}, N^{\prime \prime}, N^{\prime \prime}$-pentaacetic dianhydride (DETA- a)

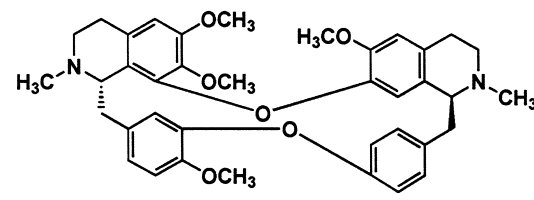

b)

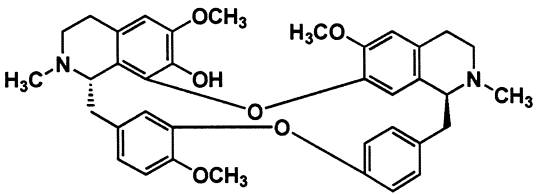<smiles>COc1cc2c(cc1O)[C@H]1Cc3ccc(OC)c(O)c3CN1CC2</smiles>

Fig. 1. Chemical Structures of the Three Alkaloids

a) TET; b) FAN; c) CYC. 
PAC; Wako Pure Chemical Industries, Tokyo, Japan) and $50 \mu \mathrm{l}$ of prepared sample were put into a test tube. After adding $50 \mu \mathrm{l}$ of $0.4 \mathrm{U} / \mathrm{ml}$ XOD (Roche, Indianapolis, IN, U.S.A.) and quick mixing, $200 \mu \mathrm{l}$ of the mixture was transferred to a flat quartz ESR cuvette, which was fixed to the cavity of an ESR spectrometer (JES-FR30, JEOL, Tokyo, Japan). Recordings of the spectra were made at $24^{\circ} \mathrm{C}$ and started at $1 \mathrm{~min}$ after the mixing of XOD; each scan took 1 min. Data were expressed as the ratio of the peak of the DMPO-OOH signal to the peak of the intrinsic standard, $\mathrm{MnO}(\mathrm{S} / \mathrm{M})$. The scavenging activity ( $\mathrm{Scv}$ ) was calculated by the following equation:

$$
\mathrm{ScV}(\%)=\left(\mathrm{S} / \mathrm{M}_{\mathrm{BLANK}}-\mathrm{S} / \mathrm{M}_{\mathrm{SAMPLE}}\right) / \mathrm{S} / \mathrm{M}_{\mathrm{BLANK}} \times 100
$$

where $\mathrm{S} / \mathrm{M}_{\mathrm{BLANK}}$ is the intensity of the ESR spectrum of DMPO-OOH spin adduct in PBS as a blank, and S/M $\mathrm{M}_{\text {SAMPLE }}$ is the intensity of the ESR spectrum of DMPO-OOH spin adduct in the sample.

$\mathrm{HO} \cdot$ was generated by the Fenton reaction ${ }^{7)}$ consisting of $75 \mu \mathrm{l}$ of $0.1 \mathrm{mM} \mathrm{H}_{2} \mathrm{O}_{2}, 50 \mu \mathrm{l}$ of each sample, $20 \mu \mathrm{l}$ of $92 \mathrm{~mm}$ DMPO and $75 \mu \mathrm{l}$ of $0.1 \mathrm{~mm} \mathrm{FeSO}_{4}$. The spectrum of DMPO$\mathrm{OH}$ was measured at $1 \mathrm{~min}$ after the addition of $\mathrm{H}_{2} \mathrm{O}_{2}$. Scavenging activity was calculated in the same manner as described above.

In Vitro Assay for Anti-hemolytic Activity of ST (ST.E, TET, FAN and CYC) Eight male Wistar rats $(350 \mathrm{~g})$ were fed commercial feed (type CE-2, CLEA Japan Inc., Tokyo, Japan) for one week. Blood obtained from rats by cardiac puncture was collected into heparinized tubes. RBC was separated from plasma by centrifugation at $1500 \boldsymbol{g}$ for $20 \mathrm{~min}$. Crude RBC was then washed three times with 5 volumes of PBS. Packed RBC was thereafter suspended in 4 volumes of PBS solution.

Hemolysis of RBC mediated by 2,2-azo-bis (2-amidinopropane) dihydrochloride (AAPH) was determined as reported previously. ${ }^{8-11)}$ The addition of AAPH to the suspension of RBC causes the oxidation of lipids and proteins in the cell membrane, thereby inducing hemolysis. It is known that AAPH-induced hemolysis in RBC is a function of incubation time and proportional to the concentration of free radicals. ${ }^{12,13)}$ The inhibitory effect on RBC hemolysis is also proportional to the concentration of antioxidants in the incubation mixture. The AAPH $(200 \mathrm{~mm})$ solution was prepared with PBS.

RBC suspension ( $1 \mathrm{ml}$ ) was mixed with $1 \mathrm{ml}$ of PBS solution containing various amounts of ST.E, TET, FAN and CYC. RBC suspension ( $1 \mathrm{ml})$ mixed with $1 \mathrm{ml}$ of PBS solu-

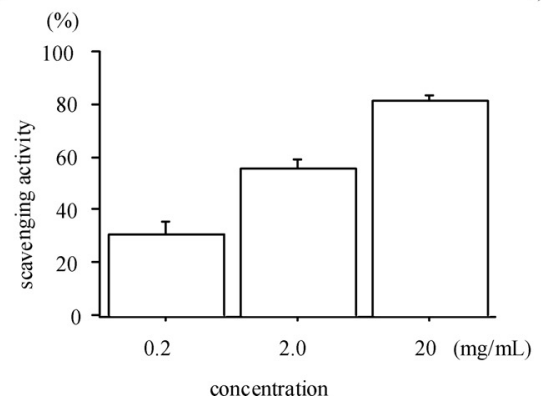

tion alone was used as control sample. The AAPH solution $(1 \mathrm{ml})$ was then added to the mixture. The incubation mixture was shaken gently in a water bath at $37^{\circ} \mathrm{C}$ for $3 \mathrm{~h}$. After incubation, $4 \mathrm{ml}$ of PBS solution was added to the reaction mixture, followed by centrifugation at $1000 \boldsymbol{g}$ for $10 \mathrm{~min}$. Absorbance (A) of the supernatant at $540 \mathrm{~nm}$ was recorded with a UV-visible recording spectrophotometer (UV-265FS, Shimadzu, Kyoto, Japan). Percentage inhibition was calculated in the same way as in the ESR assay.

Assay for Anti-hemolytic Activity of Rat Plasma after Ingestion of ST.E Twelve male Wistar rats $(350 \mathrm{~g})$ were fed the same commercial chow as for the in vitro assay for one week. They were randomly divided into two groups. After an overnight fast, one group (group A) was gavagedosed with $2 \mathrm{ml}$ of distilled water containing $200 \mathrm{mg}$ of ST.E, whereas the control group (group B) was gavage-dosed with $2 \mathrm{ml}$ of distilled water only. Thirty min after administration, blood was collected from the heart in heparinized tubes. RBC was separated from plasma by centrifugation at $1500 \mathrm{~g}$ for $20 \mathrm{~min}$. After removal of white blood cells and platelets, the remaining $\mathrm{RBC}$ was mixed with the volume of plasma therefrom. The reconstituted blood $(1 \mathrm{ml})$ was then used for the hemolysis assay by adding $1 \mathrm{ml}$ of AAPH solution and 1 $\mathrm{ml}$ of PBS followed by incubation at $37^{\circ} \mathrm{C}$ for 2,3 or $4 \mathrm{~h}$. PBS solution $(4 \mathrm{ml})$ was added to the incubation mixture followed by centrifugation at $1000 \boldsymbol{g}$ for $10 \mathrm{~min}$. Absorbance (A) of the supernatant at $540 \mathrm{~nm}$ was measured. The percentage inhibition of a gavage dose of ST.E was calculated in the same way as in the in vitro study:

This study conformed to the guidelines for the care and use of animals as approved by the Ethics Committee for Animal Experiments of Toyama Medical and Pharmaceutical University.

Statistical Analysis Data were expressed as mean \pm S.D. Repeated measures ANOVA and repeated measures one-way ANOVA followed by Fisher's PLSD were used for statistical analysis. A value of $p<0.05$ was accepted as statistically significant.

\section{RESULTS}

The $\mathrm{O}_{2}^{-} \cdot$ and $\mathrm{HO} \cdot$ scavenging activities of ST.E determined by ESR method are shown in Fig. 2. ST.E inhibited free-radical generation in a dose-dependent manner. The $\mathrm{IC}_{50}$ values for $\mathrm{O}_{2}^{-} \cdot$ and $\mathrm{HO} \cdot$ were 1.19 and $2.89 \mathrm{mg} / \mathrm{ml}$, respectively.

The anti-hemolytic effect of ST.E is shown in Fig. 3. The

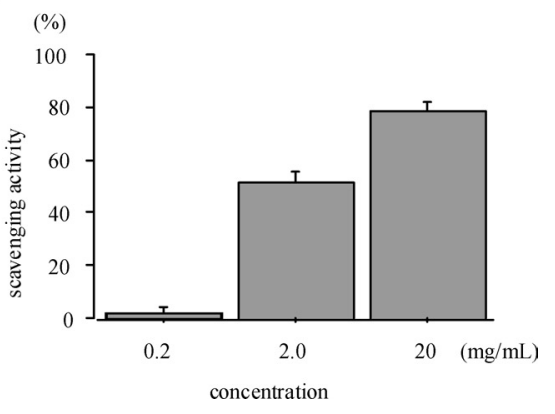

Fig. 2. ROS Scavenging Activities of ST.E

a) $\mathrm{O}_{2}^{-} \cdot$; b) $\mathrm{HO} \cdot$. Data are expressed as mean \pm S.D. of $n=4$ samples. 
water extract of ST in vitro inhibited the hemolysis of rat $\mathrm{RBC}$ due to AAPH-induced peroxy radicals in a dose-dependent manner.

Figure 4 shows the anti-hemolytic activities of the three alkaloids obtained from the methanol extract of ST. The inhibitory activity of CYC was significantly stronger than those of TET and FAN. However, the inhibition of TET at a concentration of $100 \mu \mathrm{M}$ was greater than that at $200 \mu \mathrm{M}$, suggesting that TET attains cytotoxicity at high concentration.

The results of the in vivo tests are presented in Fig. 5. RBC in reconstituted blood obtained from group A rats was more resistant to AAPH-induced hemolysis than that obtained from group B rats (Fig. 5). Together with the findings of the in vitro study, these results suggest that $\mathrm{ST}$ as antioxidant inhibits AAPH-induced hemolysis of RBC both in vitro and in vivo.

\section{DISCUSSION}

The biomembrane may be most susceptible to free-radical attack due to its content of polyunsaturated fatty acids. The present study revealed that ST.E protects these polyunsaturated fatty acids from oxidation in the membrane of RBC incubated with AAPH. Of our particular interest was the fact that the AAPH-induced lysis of human RBC was more sensitive than the ESR method in the determination of the ROS scavenging activity of ST. The mechanism, however, has still to be clarified. It has been reported that antioxidant enzyme such as superoxide dismutase, catalase, peroxidase and glutathione peroxidase were elevated in RBCs treated with $m$ chloroperbenzoic acid, a novel photosensitizer. ${ }^{14)}$ Tests are currently being planned to determine whether ST affects the

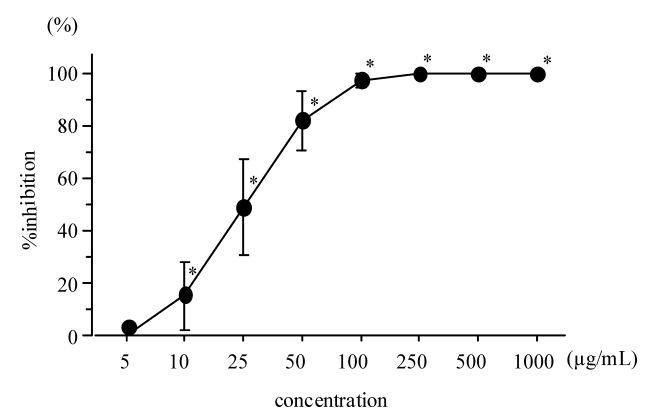

Fig. 3. Inhibitory Effect of ST.E on AAPH-Induced Lysis of Rat Red Blood Cells

Data are expressed as mean \pm S.D. of $n=8$ samples. $* p<0.0001 v$ s. control. antioxidant activities of these enzymes or not.

Although seventeen alkaloids in ST are known, ${ }^{15}$ ) their amounts are far from sufficient except for the three alkaloids, TET, FAN and CYC, examined in this study, as shown in Fig. 1. These three different alkaloids isolated from ST protected the RBC membrane from free-radical attack. Further, in this regard, it has been reported that TET has a scavenging effect on reactive oxygen species (ROS). ${ }^{16)}$ To the best of our knowledge, the present study is the first to demonstrate that FAN and CYC in vitro protect $\mathrm{RBC}$ from free-radical-induced oxidation.

Moreover, the present study was the first to demonstrate that ST in vivo augments the antioxidative effect of plasma.

It has been reported that TET and FAN have other actions, such as inhibition of $\mathrm{Ca}^{2+}$ influx, ${ }^{17,18)}$ vasodilating and hypotensive effects ${ }^{19)}$ and inhibition of platelet aggregation. ${ }^{20)}$ Together with those previously reported findings, the direct protection of RBC membrane from free-radical attack as observed in the present study would provide an important pathophysiological basis for putting to use the helpful hemorrheological, anti-atherosclerotic and anti-inflammatory effects of TET, FAN, CYC and ST.

Acknowledgments We are very thankful to Ms. Shinobu Takagi and Ms. Nanako Kuribayashi for technical assistance. This work was supported by a Grant-in-Aid for the 21st Century COE Program from the Ministry of Education, Culture, Sports, Science and Technology, Japan.

\section{REFERENCES AND NOTES}

1) Whitehouse M. W., Fairlie D. P., Thong Y. H., Agents Actions, 42, $123-127$ (1994).

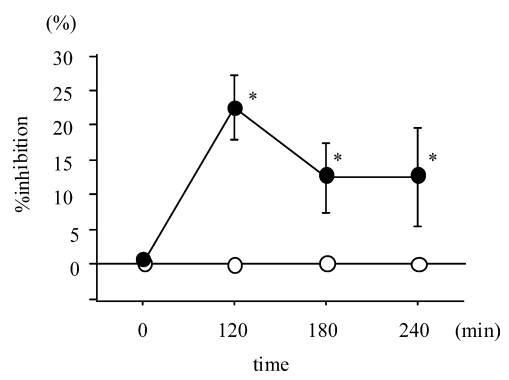

Fig. 5. The Time Course of AAPH-Induced Hemolysis of Reconstituted Blood Obtained from Rats $30 \mathrm{~min}$ after Oral Ingestion of $2 \mathrm{ml}$ of Distilled Water Containing $200 \mathrm{mg}$ ST.E (group A) or $2 \mathrm{ml}$ of Distilled Water Only (Group B)

Group A (๑), group B $(\bigcirc)$. Data are expressed as mean \pm S.D. of $n=6$ rats. Asterisks indicate significant difference from control group $(p<0.01)$.
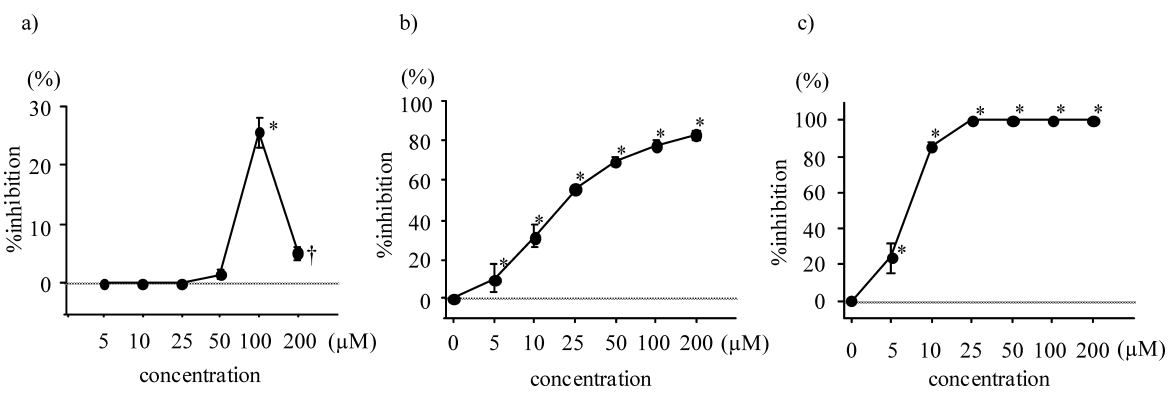

Fig. 4. Inhibitory Effect of the Three Alkaloids, the Main Ingredients of ST, on AAPH-Induced Lysis of Rat Red Blood Cells a) TET; b) FAN; c) CYC. Data are expressed as mean \pm S.D. of $n=8$ samples. ${ }^{\dagger} p<0.001, * p<0.0001 v$ s. control. 
2) Chen F., Sun S., Kuhn D. C., Lu Y., Gaydos L. J., Shi X., Demers L. M., Biochem. Biophys. Res. Commun., 231, 99-102 (1997).

3) Chang Z. M., Chang W. Y., Kuo S. Y., Chang M. L., J. Rheumatol., 24, 436- 441 (1997).

4) Ohsugi M., Fan W., Hase K., Xiong Q., Tezuka Y., Komatsu K., Namba T., Saitoh T., Tazawa K., Kadota S., J. Ethanopharmacol., 67, 111-119 (1999).

5) Buettner G. R., Free Radic. Biol. Med., 3, 259-303 (1987).

6) Mitsuta K., Mizuta Y., Kohno M., Hiramatsu M., Mori A., Bull. Chem. Soc. Jpn., 63, 187-191 (1990).

7) Kohno M., Yamada M., Mitsuta K., Mizuta Y., Yoshikawa T., Bull. Chem. Soc. Jpn., 64, 1447-1453 (1991).

8) Sekiya N., Goto H., Shimada Y., Terasawa K., Phytother. Res., 16, $373-376$ (2002).

9) Sekiya N., Shimada Y., Shibahara N., Takagi S., Yokoyama K., Kasahara Y., Sakakibara I., Terasawa K., Phytomedicine, 9, 636-640 (2002).

10) Sekiya N., Shibahara N., Sakakibara I., Hattori N., Goto H., Terasawa
K., Phytother. Res., 17, 147-151 (2003).

11) Sekiya N., Goto H., Shimada Y., Endo Y., Sakakibara I., Terasawa K., Phytother. Res., 17, 160-162 (2003).

12) Miki M., Tamai H., Mino M., Yamamoto Y., Niki E., Arch. Biochem. Biophys., 258, 373-380 (1987).

13) Sugiyama H., Fung K. P., Wu T. W., Life Sci., 53, 39-43 (1993).

14) El-Missiry M. A., Abou-Seif M., Cancer Lett., 158, 155-163 (2000).

15) Ogino T., Sato T., Sasaki H., Sugama K., Okada M., Mitsuhashi H., Maruno M., Natural Med., 52, 124-129 (1998).

16) Cao Z. F., Planta Med., 62, 413 - 414 (1996).

17) Wu S., Yu X. C., Shan J., Wong T. M., Chen C. F., Pang K. T., Life Sci., 68, 2853-2861 (2001).

18) Yu X. C., Wu S., Wang G. Y., Shan J., Wong T. M., Chen C. F., Pang K. T., Life Sci., 68, 2863-2872 (2001).

19) Kim H. S., Zhang Y. H., Oh K. W., Ahn H. Y., J. Ethnopharmacol., 58, $117-123(1997)$

20) Kim H. S., Zhang Y. H., Yun Y. P., Planta Med., 65, 135-138 (1999). 